\title{
INICIATIVAS DE PROMOÇÃO DAS DISCUSSÕES DE GÊNERO E DIVERSIDADE SEXUAL NO CONTEXTO ACADÊMICO: UM ESTUDO COMPARATIVO
}

\author{
INITIATIVES TO PROMOTE DISCUSSIONS ON GENDER AND SEXUAL DIVERSITY \\ IN THE ACADEMIC CONTEXT: A COMPARATIVE STUDY
}

\author{
INICIATIVAS PARA PROMOVER DISCUSIONES SOBRE GÉNERO Y DIVERSIDAD \\ SEXUAL EN EL CONTEXTO ACADÉMICO: UN ESTUDIO COMPARATIVO
}

\author{
Alfrancio Ferreira Dias (iD \\ Universidade Federal de Sergipe - Brasil \\ José Paulo Gomes Brazão \\ Universidade da Madeira - Portugal
}

\begin{abstract}
Resumo: Nesse artigo, apresentamos resultados parciais da pesquisa intitulada "Vozes dos estudantes universitários sobre a diversidade sexual e de gênero, sua relação com a coeducação e com a inovação pedagógica: um estudo comparativo na Universidade da Madeira (Portugal) e na Universidade Federal de Sergipe (Brasil). A partir de um realizado, apresentamos algumas reflexões acerca da inclusão de gênero e da diversidade sexual na formação docente desenvolvida pela UFS, no Brasil e UMa, em Portugal. Metodologicamente, adotamos uma abordagem qualitativa, com aplicação de questionário com quesões abertas. Tensionamos os motivos pelos quais os participantes da pesquisa consideram importante participar das/em iniciativas de inclusão de gênero e da diversidade sexual no meio acadêmico, bem como sugestões de iniciativas a desenvolver num tempo imediato, médio alcance e longo alcance. Por fim, as argumentações analisadas dos egressos evidenciam diferenças entre a formação dada pela UMa e UFS no que se refere a inclusão das discussões de gênero e diversidade sexual no meio acadêmico, apesar de identificar também diferências entre essas práticas no curso do campus de Itabaiana e em São cristóvão. Consideramos ser cedo para emitir opiniões precisas quanto a essa questão, mas esperamos que ao concluir a análise de todas as categorias da investigação, seja possível ensaiar problematizações acerca dessas diferenças.
\end{abstract}

Palavras-chave: Inclusão; Diversidade; Educação; Gênero.

Abstract: In this article, we present partial results of the research entitled "Voices of university students on sexual and gender diversity, its relationship with co-education and pedagogical innovation: a comparative study at the University of Madeira (Portugal) and at the Federal University of Sergipe (Brazil). Based on one carried out, we present some reflections on the inclusion of gender and sexual diversity in teacher training developed by UFS, in Brazil and UMa, in Portugal. Methodologically, we adopted a qualitative approach, applying a questionnaire with open questions. We stress the reasons why the research participants consider it important to participate in/in initiatives to include gender and sexual diversity in the academic environment, as well as suggestions for initiatives to be developed in an immediate, medium-range and long-range time. Finally, the arguments analyzed by the graduates show differences between the training given by UMa and UFS regarding the inclusion of discussions on gender and sexual diversity in the academic environment, despite also identifying diferences between 
these practices in the course on the campus of Itabaiana and in Saint Kitts. We believe it is too early to issue precise opinions on this issue, but we hope that by completing the analysis of all categories of the investigation, it will be possible to rehearse problematizations about these differences.

Keywords: Inclusion; Diversity; Education; Gender.

Resumen: En este artículo presentamos resultados parciales de la investigación titulada "Voces de estudiantes universitarios sobre la diversidad sexual y de género, su relación con la coeducación y la innovación pedagógica: un estudio comparativo en la Universidad de Madeira (Portugal) y en la Universidad de Sergipe (Brasil). A partir de uno realizado, presentamos algunas reflexiones sobre la inclusión de la diversidad de género y sexual en la formación docente desarrollada por UFS, en Brasil y UMa, en Portugal. Metodológicamente, adoptamos un enfoque cualitativo, aplicando un cuestionario con preguntas abiertas. Destacamos las razones por las cuales los participantes de la investigación consideran importante participar en / en iniciativas para incluir la diversidad de género y sexual en el ámbito académico, así como sugerencias de iniciativas a desarrollar en un tiempo inmediato, mediano y largo. Finalmente, los argumentos analizados por los egresados muestran diferencias entre la formación impartida por la UMa y la UFS en cuanto a la inclusión de discusiones sobre género y diversidad sexual en el ámbito académico, a pesar de identificar también diferencias entre estas prácticas en el curso en el campus de Itabaiana y en Saint Kitts. Creemos que es muy pronto para emitir opiniones precisas sobre este tema, pero esperamos que al completar el análisis de todas las categorías de la investigación, sea posible ensayar problematizaciones sobre estas diferencias.

Palabras clave: inclusión; Diversidad; Educación; Género.

\section{Algumas considerações iniciais}

As discussões de gênero estão sendo considerados na literatura recente como um elemento das relações de poder e nas circunstâncias de flutuantes e rizomáticas da contemporaneidade. No campo da Educação, urge um olhar dissidente, não normativo, enquanto ato político acerca das questões de gênero e da sexualidade, conforme influências dos estudos pós-identitários.

Dias e Amorim (2015) sistematizaram a literatura específica sobre corpo, gênero, sexualidades e formação docente, identificando que, independente do tipo de pesquisa utilizado, uma característica é comum entre os estudos analisados relativos às temáticas: o fato de que todos (com maior ou menor grau de intensidade) sugerem que a abordagem das temáticas contribui para a desestabilização de normatizações, classificações e hierarquizações no campo da educação. Esse resultado também foi evidenciados nas pesquisas de Dias, Oliveira e Matos (2018), Silva, Dias e Rios (2020) e Dias, Silva e Rios (2020).

Cardoso, Bertoldo e Santos (2020) analisaram as dissertações e teses em gênero, sexualidade e formação docente defendidas em programas de pós-graduação nas instituições de ensino superior das regiões Norte e Nordeste do Brasil entre os anos de 2006 a 2018 . As autoras, evidenciaram a partir da análise das pesquisas desenvolvidas que os "debates e 
pesquisas voltadas para as temáticas de gênero e sexualidade não garantem que os/as professores/as em formação ou em experiências formativas continuadas mudem comportamentos e discursos” (p. 1759). Contudo, há evidências de que elas também "possibilitam mais espaços de discussões tanto na formação inicial quanto na continuada" (p. 1759). O que sugere que estamos em espaços de novas posturas e não criando outros enquadramentos (DIAS; MENEZES, 2017).

Dal'igna, Scherer e Cruz (2017) analisaram a produção acadêmica desenvolvida por pesquisadores e pesquisadores vinculados ao GT 23: Gênero, Sexualidade e Educação da ANPED e refletiram sobre os "modos como gênero e sexualidade são abordados ao focalizar a formação de professores, e oferece contribuições para que se possa construir e delimitar futuros problemas de pesquisa sobre esses temas" (p. 632). As autoras destacam que ainda "há muito o que fazer/dizer/investigar sobre gênero, sexualidade e formação de docentes em nosso país" (p. 648).

De fato, há muito o que problematizar sobre gênero e sexualidade na formação de professores e professoras. Consideramos importante destacar que esses estudos que destacamos evidenciam um forte crescimento do produção científica na área da educação (FERREIRA, 2015; 2017; FERREIRA, 2013; GUARANY; SANTOS; CARDOSO, 2020; DIAS; AMORIM, 2015; SILVA; DIAS; RIOS, 2020; CARDOSO; DIAS, 2021; GUARANY; CARDOSO, 2021; SILVA; RIOS, 2020; MEDEIROS; SANTOS, 2020), o que nos oferece um panorama das inovações e principais resultados e contribuições dos estudos para a produção de políticas públicas, ampliação e sensocumonização da temática nos cursos de formação docente, bem como um contradiscurso ao avanço de conservadorismo e abordagens reacionárias que propagam um discurso de ódio por meio da chamada "ideologia de gênero"1.

Conforme Carvalho et al. (2017), a inclusão da perspectiva da diversidade sexual e de gênero na educação e na formação docente é urgente, visto que os estudos de gênero e sexualidade ainda não foi foi incluído fortemente no "pensamento educacional no Brasil, nem se transversalizou na educação superior, nem na formação docente inicial (cursos de Pedagogia e formação de professores/as), nem na escola (na prática pedagógica e curricular e na formação docente continuada), apesar do direcionamento das políticas públicas recentes (CARVALHO, et al., 2017, p. 50). Nesse sentido, faz necessário mais investigações que analisem as discussões

\footnotetext{
${ }^{1}$ Segundo Furlani (2015), a "Ideologia de Gênero" foi discurso inventado, especialmente, no interior de alguns discursos religiosos. Trata-se de uma interpretação, equivocada e que não reflete o entendimento de "gênero" presente na educação, nas práticas educativas e cursos de formação doente. Para saber mais sobre essa discussão, recomendamos a leitura dos trabalhos de Misckolci e Campana (2017), Misckolci (2018), Junqueira (2018) e Kalil (2019).
} 
de gênero, sexualidades e outros marcadores sociais da diferença no campo da educação e na formação docente.

Nossa intenção com essa investigação foi contribuir para a discussão da áera dos estudos de gênero e educação, a partir dos resultados do projeto de pesquisa de estágio de pós-doutorado do segundo autor e supervisionada pelo segundo autor, intitulada "Vozes dos estudantes universitários sobre a diversidade sexual e de gênero, sua relação com a coeducação e com a inovação pedagógica: um estudo comparativo na Universidade da Madeira (Portugal) e na Universidade Federal de Sergipe (Brasil)"2. Com o seu desenvolvimento, pretendemos numa perspectiva comparativa, refletir sobre a diversidade sexual e de gênero no meio acadêmico, refletindo sobre ambientes inclusivos e o seu contributo no campo da inovação pedagógica. Tensionamos a discussão destes temas na academia, a fim de problematizar e contribuir para a renovação conceitual e dos contextos organizacionais da prática da pedagogia (BRAZÃO; OLIVEIRA; DIAS, 2021; PALMEIRA; DIAS, 2021; CARDOSO; DIAS, 2021; OLIVEIRA; BRAZÃO).

Anteriormente, Rios, Cardoso e Dias (2018) realizaram uma investigação com o objetivo de refletir acerca das concepões dos docentes do curso de licenciatura em Pedagogia da Universidade Federal de Sergipe, campus de Itabaiana, sobre as temáticas de gênero, corpo e sexualidade. Os autores, sinalizaram perceptíveis avanços em relação às temáticas abordadas, a partir das implicações epistemológicas de alguns docentes. A pesquisa também apontou a necessidade de uma mudança na estrutura curricular, incorporando as temáticas e posturas de uma pedagogia queer que contribua para a promoção de novas posturas políticas, estratégias, atitudes, procedimentos pedagógicos subversivos e de negociação na universidade.

Ainda sobre o contexto do curso de Pedagogia da UFS, Pires (2021) analisou como os cursos de Pedagogia da Universidade Federal de Sergipe, campus de São Cristóvão e de Itabaiana elaboram propostas curriculares que inclusão às questões de gênero e sexualidade, bem como que visibilidade as temáticas possuem e, em especial, qual profissional pretende formar e para que atuação. A autora identificou diferentes perspectivas de currículo e de abordagens das temáticas, com fortes avanços no curso de Pedagogia do campus de Itabaiana, na medida em que o currículo como "produtor de subjetividades e permeado por relações de poder, e as discussões sobre gênero e sexualidade passam a ser contempladas de forma significativa, demonstrando disposição em ampliar a problematização e compreensão da relação entre a educação e a complexidade social" (PIRES, 2021, p. 04). Apesar dos avanços apontados, a autora evidencia a existência nos currículos dos dois cursos investigados de

\footnotetext{
${ }^{2}$ Ver mais informações em Brazão (2021), em https://bra.in/7vA6Q3
} 
concepções diversas de "educação, gênero e sexualidade subjetivadas pelas políticas públicas identitárias neoliberais que buscam o consensual, normalizar e homogeneizar" (PIRES, 2021, p. 04$)^{3}$.

No contexto da Universidade da Madeira, destacamos a investigação realizada por Mendonça et al. (2019), na qual analisou as representações de estudantes da formação de professores em educação infantil sobre estereótipos de gênero. Os autores identificaram que as discussões de gênero "se encontra limitada a duas Unidades Curriculares e que, entre os estudantes, subsiste a ideia de que a feminização daquela profissão se encontra de tal modo enraizada que a implementação de quotas de género dificilmente conseguirá inverter esta tendência" (p. 96). O estudo ainda concluiu que pedagogicamente, "a diversidade e a heterogeneidade foram elencadas como fatores que permitem aumentar mais a produtividade das crianças" (p. 96) e que na socialização, "a divisão de tarefas entre os géneros acentua-se com o aumento da idade e em situação conjugal” (p. 6).

As pesquisas de Rios, Cardoso e Dias (2018), Pires (2021) no contexto da UFS e a de Mendonça et al. (2019) no contexto da UMa são significativas para as problematizações sobre como os cursos de formação docente estão discutindo as questões de gênero e sexualidade. Assim, nossa intenção com a pesquisa ${ }^{4}$ foi contribuir para o avanço dos estudos de gênero, a partir da análise das concepções dos egressos sobre gênero e diversidade sexual das duas universidades, bem como espaços e práticas dissidentes ou de inovação pedagógica no tocante ao trabalho pedagógico com a stemáticas. Para esse artigo, informamos ao leitor que realizamos um recorte dos dados coletados da pesquisa, focando na reflexão acerca da inclusão de gênero e da diversidade sexual na formação docente desenvolvida pela UFS, no Brasil e UMa, em Portugal.

\section{Metodologia da investigação}

Utilizámos na pesquisa uma abordagem metodológica qualitativa de natureza exploratória (NASCIMENTO; CAVALCANTE, 2018; NUNES, 2020; ALVES; FIALHO; LIMA, 2018) para fundamentar os fundamentos dos processos de coleta de dados, em especial, porque as investigações sobre os estudos de gêneros e outros marcadores da diferença, dada sua rica natureza subjetiva. Por se tratar de uma investigação com densidade e varidade de dados, para nesse artigo iremos apenas apresentar a estudo comparativo das enunciações dos egressos

\footnotetext{
${ }^{3}$ Aos leitores podem saber mais sobre essa perspectivas nos trabalhos de Cardoso, Rios e Dias (2018), Dias, et al. (2017) e Dias (2015).

${ }^{4}$ Ver mais detalhes sobre a investigação em Brazão, Oliveira e Dias (2021).
} 
acerca da inclusão de gênero e diversidade sexual, em dois contextos universitários: Universidade Federal de Sergipe e Universidade da Madeira.

Os questionários que serviram ao levantamento dos dados nesta categoria mantiveram o mesmo número de questões, tendo o texto sido adaptado com expressões linguísticas aos dois contextos estudados. Apresentamos duas questões de resposta fechada, a primeira, sobre a importância que atribuíam à inclusão da diversidade sexual e de gênero, no contexto acadêmico e a segunda, sobre a participação dos estudantes nessa ação. Posteriormente, colocamos quatro questões abertas sobre as iniciativas desenvolvidas no contexto acadêmico sobre inclusão da diversidade sexual e de gênero e sobre as sugestões para futuras iniciativas neste âmbito. Os questionários encontram-se acessíveis nos links abaixo especificados ${ }^{5}$.

Utilizamos para recolha de informação o Google Forms da Google Drive resources. Definimos dois grupos de amostra de conveniência: a) os egressos do curso de mestrado em Educação Pré-Escolar e Ensino do $1^{\circ}$ Ciclo do Ensino Básico, da UMa, entre 2015 e 2020. Solicitamos o preenchimento a 160 ex-estudantes da Universidade da Madeira. Destes apenas obtivemos 22 respostas (13,7\%); b) os egressos dos cursos de graduação em Pedagogia da UFS, entre 2015 e 2020, do Campus de São Cristóvão e do Campus de Itabaiana. Solicitamos o preenchimento dos questionários preenchimento a 183 ex-estudantes da Universidade Federal de Sergipe e obtivemos 26 respostas ( $14,21 \%)$.

Os dados qualitativos foram analisados com o auxílio de um programa informático que elaboramos para executar a análise de conteúdo das categorias estudadas (BARDIN, 2011) e que inclui a transcrição das justificações dos egressos, a construção das categorias de análise, em tabelas, ilustradas pelas unidades de significação semântica (BOGDAN; BLIKEN, 1994). Os recortes textuais foram codificados com a seguinte lógica: [País (PT ou Br) (-); campus universitário Itabaiana (ITA) ou São Cristóvão (SC); número de anos em que encontram após

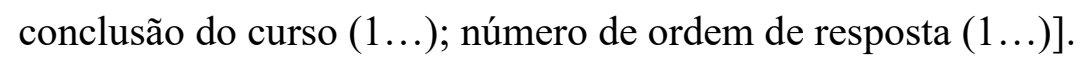

Utilizamos a ferramenta FileMaker Pro v18, elaboradora de bases de dados relacionais, da Claris International Inc., muito conhecida no meio acadêmico. Este programa informático está desenvolvido para o Windows. Para além de organizar os recortes categorizados e subcategorizados dos textos, o programa contém conexões com um módulo de interpretação dos dados, uma vez que estabelece uma relação direta entre a análise dos recortes obtidos e os referenciais teóricos, selecionados para fundamentar a interpretação dos fenómenos.

\footnotetext{
${ }^{5}$ Os questionários encontram-se acessíveis nos links:

Para os ex-estudantes da Universidade da Madeira:

https://drive.google.com/file/d/1okl-9ue088QFOy2dBtuyvQ9xXSTLmvKi/view?usp=sharing

Para os ex-estudantes da Universidade Federal de Sergipe:

https://drive.google.com/file/d/14M7EWnjiB3-yQWtFUD-0JbJKmX1YU-0Y/view?usp=sharing
} 
Figura 1 - Base de dados para análise de conteúdo dos dados qualitativos

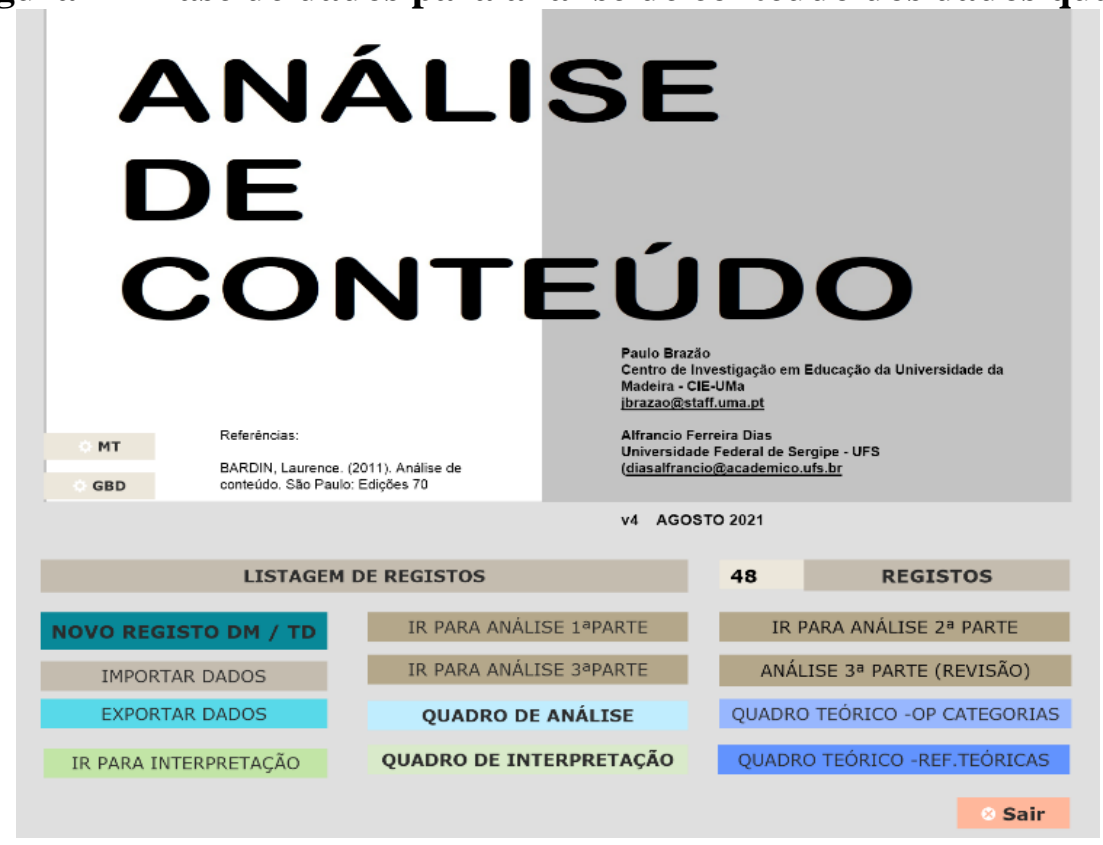

Fonte: Os autores (2021)

Os textos com as afirmações dos egressos foram arrumados por recortes unidades de significação semântica, conforme sugerem Bardin (2011) e Bogdan e Bliken (2017). Assim, a categoria analisada neste artigo, a Inclusão de gênero da diversidade sexual no meio acadêmico, deu origem a subcategorias e dentro de cada uma destas encontramos diferentes fenômenos, conforme podemos ver no quadro 1 de categorização dos resultados.

\section{Quadro 1: Categorização dos resultados}

\begin{tabular}{|c|c|}
\hline \multicolumn{2}{|c|}{ Categoria } \\
\hline \multicolumn{2}{|c|}{ INCLUSÃO GÊNERO E DIVERSIDADE SEXUAL NO MEIO ACADÊMICO } \\
\hline Subcategoria & Fenômenos \\
\hline $\begin{array}{l}\text { Motivos pelos quais os egressos consideram importante } \\
\text { participar em iniciativas de inclusão de gênero e } \\
\text { diversidade sexual no meio acadêmico. }\end{array}$ & $\begin{array}{l}\text { Promoção da inclusão enquanto direito e garantia dos } \\
\text { cidadãos, na qualidade e bem-estar social. }\end{array}$ \\
\hline $\begin{array}{l}\text { Participação dos ex-estudantes em ações sobre a inclusão } \\
\text { de gênero e diversidade sexual no meio acadêmico. }\end{array}$ & $\begin{array}{l}\text { Iniciativas livres sobre inclusão de gênero e diversidade sexual } \\
\text { no meio acadêmico. } \\
\text { Participação dos egressos em ações estruturadas sobre a } \\
\text { inclusão de gênero e diversidade sexual no meio acadêmico. }\end{array}$ \\
\hline $\begin{array}{l}\text { Motivos pelos quais não participaram em iniciativas de } \\
\text { inclusão de gênero e diversidade sexual no meio } \\
\text { acadêmico. }\end{array}$ & $\begin{array}{l}\text { Dificuldade em promover a inclusão de gênero e diversidade } \\
\text { sexual no meio acadêmico. } \\
\text { Motivos pessoais. } \\
\text { Não existência de eventos na instituição acadêmica sobre o } \\
\text { tema. }\end{array}$ \\
\hline \multicolumn{2}{|l|}{$\begin{array}{l}\text { Referências à não necessidade de promover futuras } \\
\text { iniciativas sobre a inclusão de gênero e diversidade sexual } \\
\text { no meio acadêmico. }\end{array}$} \\
\hline $\begin{array}{l}\text { Sugestões de iniciativas futuras sobre a inclusão de gênero } \\
\text { e diversidade sexual no meio acadêmico. }\end{array}$ & $\begin{array}{l}\text { Iniciativas a desenvolver num tempo imediato. } \\
\text { Iniciativas a desenvolver a médio alcance. } \\
\text { Iniciativas a desenvolver a longo alcance. }\end{array}$ \\
\hline
\end{tabular}

Fonte: Os autores (2021) 


\title{
Discussão dos resultados
}

\begin{abstract}
Para quebrar tabus, informar e conscientizar cada vez mais as pessoas sobre respeito e que não existe padrões quando o assunto é o outro. Deve-se, de uma vez por todas, acabar com o preconceito e o entendimento de que se pode decidir pelo outro, pois não pode. Por isso se faz importante essa inclusão, para que também todos se sintam livres, acolhidos, importantes e inclusos na sociedade (BR-ITA-3-03).
\end{abstract}

Problematizar, concientizar, quebrar tabus, acabar, decidir, sentir, liberdade, acolher. Nossa! Quanta potência. Um convite a refletir: "não existe padrões quando o assunto é o outro" ou "decidir pelo outro, pois não pode". Um potente convite a desaprender. A participante, certamente está nesse processo. E nós? E vocês? Tentando? Nossa intenção é de tencionar e propor reflexões sobre a inclusão das discussões de gênero e diversidade sexual, pois com sentir-se livre e acolhido é um bom começo para o ser e estar na escola e ou universidade. "Informar cade vez mais", nos diz a participante. Discutir, sentir, ouvir! Um movimento outro. Contrário à normalização dos corpos, do controle, da criação de expectativa sobre o outro. Fazse importante incluir as temáticas, problematizando esteriótipos, preconceitos e normalizações.

Nessa perspectiva, iniciamos nossa discussão com os motivos pelos quais os participantes da pesquisa consideram importante participar das/em iniciativas de inclusão de gênero e da diversidade sexual no meio acadêmico. Nesta subcategoria, encontramos o fenômeno da promoção da inclusão enquanto direito e garantia dos cidadãos, na qualidade de vida e bem estar social (45) que agrupa as respostas dos egressos da UMa (22) respostas, e dos egressos da UFS de Itabaiana (16) respostas, e com menos frequência no campos das justificativas dos participantes da UFS de São cristóvão (6) respostas. Vejamos alguns argumentos apresentados pelos participantes:

Enquanto pessoas e profissionais da área de educação precisamos trabalhar com e para a diversidade, tanto no acolhimento, apoio e comprometimento em prol do respeito e direito das pessoas LGBTQIA+ (BR-SC-5-02).

A educação é uma importante ferramenta de inclusão e promoção de direitos, o importante usar ela para diminuir a LGBTIfobia no Brasil (BR-SC-1-01).

É importante que nossas crianças não cresçam com medo de serem reais e que elas passem a vida sentindo-se incomodadas com o seu sexo ou corpo por falta de conhecimento sobre (BR-ITA-5-17).

Sim, pois incentivaria as crianças a terem mais respeito e empatia pelas pessoas (BRITA-5-16).

É um tema discutido mundialmente, que pode modificar a cultura preconceituosa que está impregnada em nossa sociedade (BR-ITA-5-14).

É fundamental para que as pessoas possa tem mais conhecimento e a partir do conhecimento entendam que todos tem direitos iguais e que precisa ser respeitados por todos (BR-ITA-5-13). 
Poderemos quebrar um pouco com esse tabu preconceituoso, que acaba fazendo com que muitas pessoas deixem de ser felizes por medo da sociedade, que tornando isso mais difícil, promovendo mais ódio, pois quando não há informações tudo torna-se difícil. (BR-ITA-4-10)

Para sabermos ter uma opinião de cada gênero e o principal saber diferenciar e respeitar essas pessoas (BR-ITA-3-07).

Quanto mais pessoas conhecerem a importância da inclusão da diversidade sexual, será aceita e conhecida, menos discriminação e mais aceitação (BR-ITA-3-05).

Para que as pessoas conheçam as diversas formas de gênero, priorizando o respeito e a igualdade (BR-ITA-3-04).

Acho importante para que tenhamos cada dia uma sociedade mais sábia e menos preconceituosa (BR-ITA-3-02).

Para libertar de conceitos pré-concebidos à luz de valores e ideias erradas (PT-5-21).

É urgente consciencializar e abordar esta questão desde o mais cedo possivel e principalmente em contexto académico. Etapa onde definimos qual o papel que queremos ter em sociedade (PT-5-18).

É importante promover a inclusão da diversidade sexual e de género para que não haja discriminação (PT-3-12).

Num contexto de divulgar de forma simples e educar a sociedade para a diversidade (PT-3-11).

Muito importante para nos sentirmos confiantes com as nossas escolhas (PT-1-06).

Vivemos num mundo em constante mudança, onde estas questões estão cada vez mais evidentes na nossa sociedade. Para uma evolução das mentalidades é imprescindível consciencializar o ser humano (PT-1-05).

Urge a necessidade de alargar o conhecimento acerca destes temas e é fundamental combater a discriminação que se faz sentir em relação aos mesmos (PT-1-01).

Os participantes (BR-SC-5-02) e (BR-SC-1-01) consideram o trabalho pedagógico com as temáticas como uma ferramenta de promoção de espaços inclusivos e de diminuição de ações discriminatórias com estudantes LGBTQI+. Esse argumento foi acionado por mais de $50 \%$ dos participantes, bem como entre os que destacamos de Itabaiana da UFS e da UMa (BR-ITA-514; BR-ITA-4-10; BR-ITA-5-13; BR-ITA-3-05; BR-ITA-3-02; PT-3-12; PT-1-01), bem como é apresentado em outros estudos que evidenciam que o trabalho pedagógico com as temáticas potencializa a diminuição a LGBTIfobia e desconstruções de esteriótipos (ALMEIDA, 2017; BOGOSSIAN, 2014; CARDOSO, 2019; CARVALHO et al., 2017; COUTO; CRUZ, 2017; DONATO; TONELLI, 2018; FRANÇA; FERRARI, 2016; MORAIS; BAIÃO; DE FREITAS, 2020; SANTOS; RIOS, 2021)

Alguns argumentos evidenciaram que a inclusão das temáticas no contexto acadêmico colabora com o processo de desaprendizagem de concepçõees de gênero e sexualidades mais tradicionais e potencializar formas inovadoras de educar para a diversidade (PT-5-18; BR-ITA3-05; PT-5-21; PT-3-11; PT-1-01). Essas argumentações também foram idientificados nos 
estudos de Rios, Cardoso e Dias (2018) e Pires (2021), ao analisarem das práticas educativas do curso de Pedagogia da Itabaiana, bem como nos estudos de Santos e Feldens (2019), Santos e Lage (2017), Silva Junior e Ivenicki (2019), Dias et al. (2017), Cardoso e Dias (2017) Menezes, Dias e Santos (2020), Moreira, Evangelista e Santos (2019) e Cirqueira, Santana e Pereira (2021).

Observamos nos argumentos apresentados pelos egressos de Itabaiana da UFS uma abertura ou sinais uma perspectiva de currículo mais pós-crítico (PIRES, 2021), na medida em que foram destacados a importãncia da inclusão das temáticas como facilitadoras de espaços de empatia pelas pessoas (BR-ITA-5-16), de conhecimento sobre sexualidade e sentidos do corpo (BR-ITA-5-17), conhecer gênero (BR-ITA-3-07), viver gêneros (BR-ITA-3-04), autoestima sobre as suas experiências sexuais (PT-1-06).

Sobre a subcategoria para reunir os motivos pelos quais os egressos não participaram em iniciativas de inclusão de gênero e diversidade sexual no meio acadêmico, identificamos três fenômenos: a) dificuldade em promover ações; b) motivos pessoais; c) inexistência de eventos e/ou outras ações. A dificuldade em promover ações foram destacados pelos participantes de São Cristóvão (UFS) e da UMa: “Com muito cuidado em uma escola publica mais estruturada, não na situação que se encontra nossa educação" (BR-SC-5-09); "Nunca tive oportunidade e penso que estes temas não são bem aceites pela maioria dos professores" (PT1-03); "Nunca surgiu oportunidade e são temas que não são bem aceites pela maioria dos docentes" (PT-1-01). A BR-SC-5-09 conecta as dificuldades na promoção ao momento nos últimos anos dos dicursos conservadores no Brasil e nas políticas educacionais. Contudo, os participantes PT-1-03 e PT-1-01 destacaram a rejeição por parte dos docentes quanto à discussão das temáticas. Em Portugal, desde 2011, os planos nacionais sobre as políticas públicas, integram a área estratégica da orientação sexual e identidade de género com propósito de "prevenir e combater todas as formas de discriminação em função da orientação sexual e da identidade de género e promover a sensibilização de toda a sociedade portuguesa para esta problemática." CIG-PT. Talvez isso ocorra devido ao período de formação ou a implementação de ações institucionais. A partir de 2018, Portugal passou a ter um plano autónomo intitulado "Plano de Ação de combate à discriminação em razão da Orientação sexual, Identidade e Expressão de género e Características sexuais". O governo português aprovou e implementou o projeto "Portugal + Igual", como foco em três eixos de ação: Igualdade entre homens e mulheres; Combate à violência contra mulheres e violência doméstica; Combate à discriminação em razão da orientação sexual, identidade do gênero e características sexuais 
(PORTUGAL, 2018). De fato, é um avanço significativo instituionalizar políticas como essas, mas faz-se necessário também, ampliar sua divulgação e elaborar estratégias formativas.

Sobre os motivos pessoais que os egressos justificaram a não participação em iniciativas de inclusão de gênero e diversidade sexual no meio acadêmico foram ao nível de desconforto (BR-SC-5-09), oportunidade (BR-SC-5-04; BR-ITA-5-14; BR-ITA-3-04), nunca refletiu sobre ou não teve a ideia (BR-SC-5-02; PT-4-16; PT-3-15), porque não quis (PT-5-17) ou "porque para mim é tudo considerado normal" (PT-2-08). Esses argumentos também foram identificados nos estudos de Cirqueira, Santana e Pereira (2021), Santos e Lage (2017), Silva Junior e Ivenicki (2019) Santos e Rios (2021) e Mendonça et al. (2019).

No que se refere a inexistência de eventos e/ou outras ações institucionais, destamos alguns argumentos apresentados pelos partipantes: "Não houve eventos por parte da Universidade" (BR-SC-5-05), "Não houveram momentos e espaço para que essas questões viessem a desenvolver (BR-SC-5-03), "Poucos estudos ou pesquisas que aprofundassem os temas com maior profundidade"(BR-SC-5-03), “Ainda não existia uma disciplina voltada para esse tema” (BR-ITA-3-07), "Nunca ocorreu” (PT-5-22; PT-5-22; PT-3-13; PT-1-07), “Tema não abordado. Nunca tive conhecimento de alguma iniciativa" (PT-5-18; PT-3-14; PT-1-06), "Não surgiu nenhuma oportunidade para esta participação" (PT-3-12; PT-3-10; PT-3-10).

Após problematizar os motivos pelos quais os participantes não participaram de iniciativas incluvidas das temáticas, elaboramos uma subcategoria para reunir argumentos sobre a participação dos egressos em ações sobre a inclusão de gênero e diversidade sexual no meio acadêmico. Com isso verificamos dois fenômenos: o primeiro, destaca iniciativas livres sobre inclusão das discussões de gênero e diversidade sexual no meio acadêmico, conforme trechos: "como ouvinte ou dando apoio logístico" (BR-SC-5-08), "Participei de iniciativa de inclusão de qualquer pessoa no contexto acadêmico e não um grupo em específico" (BR-SC-5-06). O segundo, as ações estruturadas inclusão das discussões de gênero e diversidade sexual no meio acadêmico: participação no Programa de Iniciação a Docência - PIBID sobre a formação docente nas temáticas de gênero, sexulaidade e raça (BR-ITA-5-13 BR-ITA-5-12; BR-ITA-305; BR-ITA-3-05; BR-ITA-4-08; BR-ITA-2-01); desenvolvimento de pesquisa de conclusão de curso acerca da análise de gênero no livros didáticos de matemática da séries iniciais do ensino fundamental da rede pública e estadual (BR-ITA-5-13); participação em grupo de pesquisa sobre gênero e sexualidade (BR-ITA-4-1; BR-ITA-3-05); Desenvolvimento de oficinas nas escolas sobre gênero e sexualidade (BR-ITA-4-10; BR-ITA-3-05; BR-ITA-3-06); aulas sobre alguns conceitos (BR-ITA-4-09; BR-ITA-4-08), palestras (BR-ITA-3-02). Nessa perspectiva, observamos que os argumentos apresentam uma diversidade de ações que o curso 
de Pedagogia da UFS Itabaiana desenvolveu ao longo do processo formativo dos participantes, conforme também evidenciam Pires (2021) e Rios, Cardoso e Dias (2018). Contudo, pode sugerir ausências dessas ações no curso de Pedagogia da UFS São Cristóvão, apesar de está situado no mesma instituição, bem como o realizade da UMa, pois não foi identificada, dentre os participantes ações tanto livres quanto estruturadas.

Por fim, elaboramos uma subcategoria para reunir as sugestões de iniciativas futuras sobre a inclusão de gênero e diversidade sexual para além do meio acadêmico. Categorizamos os daods em três fenômenos: a) referências a iniciativas a desenvolver num tempo imediato; b) referências sobre iniciativas a desenvolver num tempo de médio alcance; c) referências sobre iniciativas a desenvolver num tempo de longo alcance. No grupo das iniciativas imediatas, foram destacadas ações como: eventos, mesas redondas, palestras, diálogos e discussões abertas e exposições (BR-ITA-4-11; BR-ITA-3-07; BR-ITA-3-07; PT-5-18; PT-3-15; PT-3-14; PT-312; PT-3-13; PT-3-11; PT-2-09; PT-1-07; PT-1-05), inclusão de ações no calendário acadêmico (BR-ITA-4-09; PT-5-18; PT-1-05; PT-1-04), oficinas e aulas sobre as temáticas (BR-ITA-408; PT-1-04; PT-1-03; PT-1-01) e ações institucionais de conscientização, a fim de gerar um ambiente inclusivo, com inovações em organização estrutural e de políticas, resultantes no engajamento para uma maior segurança e inclusão no que diz respeito a diferença (BR-ITA-303).

No grupo das iniciativas com desenvolvimento médio alcance, foram sugeridas as ações: realização de pesquisas com novas abordagens para impactem nas ações de ensino (BRSC-5-03); inclusão e implementação de gênero e diversidade nos currículos educacionais (BRITA-5-13; BR-SC-1-01; BR-ITA-2-01; BR-ITA-4-10); formação de professores, possibilitando capacitação pedagógica para trabalhar com as temáticas em na sala de aula (BR-ITA-5-16; BRITA-5-14; BR-ITA-5-14; BR-ITA-5-13); elaboração de ações extensionistas para que a universidade possa contribuir com a concientização sobre as temáticas nas escolas de educação básica, contribuindo para a capacitação continuada de docentes (BR-ITA-5-14; BR-ITA-5-12; BR-ITA-3-05). No grupo das iniciativas com desenvolvimento de longo alcance foram sugeridas: formação nas temáticas em entidades sociais municiais como CRAS e CREAS (BRSC-5-09), Inclusão do tema na escola, nos currículos de formação profissional e nas políticas públicas (BR-SC-5-07); iniciativas que vão além do contexto acadêmico, como a família e outras instituições da sociedade (BR-ITA-3-02); elaboração Políticas inclusivas e Legislações que garantam a socioalizam plena de todos (PT-5-22; PT-5-21; BR-ITA-5-17).

Ao refletir acerca das sugestões dos participantes, vemos o caminho de possibilidades profícuas, em especial o da formação. Segundo Carvalho et al. (2017), ao formar gestores, 
professores, e demais profissionais da educação as temáticas podem a ser incluídas, debatidas e ressignificadas no interior no cotidiano escola e na sociedade.

\section{Conclusão}

O debate de questões sobre as relações gênero e diversidade sexual são muito atuais nos movimentos sociais. Contudo, no campo da Educação existem invariantes culturais presentes nos ambientes escolares e acadêmicos, responsáveis pela inação na mudança e na melhoria desses contextos. Estamos convictos que esta pesquisa pode contribuir para a busca de mudança de paradigma uma vez que ela se foca na reflexão de egressos sobre do conhecimento em torno de práticas inclusivas sobre as temáticas, no campo acadêmico e desenvolvimento profissional (BRAZÃO; OLIVEIRA; DIAS, 2021).

O conhecimento das enunciações dos participantes da investigação, egressos do curso de mestrado em Educação Pré-Escolar e Ensino do $1^{\circ}$ Ciclo do Ensino Básico, da Universidade da Madeira e egressos dos cursos de graduação em Pedagogia da Universidade Federal de Sergipe, permitiu conhecer como as temáticas de gênero e da diversidade sexual foram abordadas na formação docente, de forma comparada.

A partir da análise dos resultados, percebemos que os motivos pelos quais os participantes da pesquisa consideram importante participar das/em iniciativas de inclusão de gênero e da diversidade sexual no meio acadêmico foi percebida a partir do fênomero da promoção da inclusão enquanto direito e garantia dos cidadãos, na qualidade de vida e bem estar social. Também, evidenciaram que a inclusão das temáticas no contexto acadêmico colabora com o processo de desaprendizagem de concepçõees de gênero e sexualidades mais tradicionais e potencializar formas inovadoras de educar para a diversidade.

Ao refletir acerca da participação dos egressos em ações sobre a inclusão de gênero e diversidade sexual no meio acadêmico, verificamos dois fenômenos centrais a partir de iniciativas livres e as ações estruturadas inclusão das discussões de gênero e diversidade sexual no meio acadêmico. Sobre a subcategoria que reune os motivos pelos quais os egressos não participaram em iniciativas de inclusão de gênero e diversidade sexual no meio acadêmico, agrupamos os fenômenos: dificuldade em promover ações, motivos pessoais e inexistência de eventos e/ou outras ações.

Ao analisar as sugestões de iniciativas futuras sobre a inclusão de gênero e diversidade sexual para além do meio acadêmico, concluimos que os participantes sugerem referências a iniciativas a desenvolver num tempo imediato, médio alcance e longo alcance. 
Por fim, as argumentações analisadas dos egressos evidenciam diferenças entre a formação dada pela UMa e UFS no que se refere a inclusão das discussões de gênero e diversidade sexual no meio acadêmico, apesar de identificar também diferências entre essas práticas no curso do campus de Itabaiana e em São cristóvão. Consideramos ser cedo para emitir opiniões precisas quanto a essa questão, mas esperamos que ao concluir a análise de todas as categorias da investigação, seja possível ensaiar problematizações acerca dessas diferenças. Uma vez que os discursos sobre gênero dos egressos não são estáveis e que variam em função dos contextos (Pereira, 2012). Assim, nossa expectativa é possibilidades de identificação de grandes linhas de tendência onde a compreensão e a explicação se entrecruzam (Stake, 2009) possibilitando ainda significâncias dos seus discursos.

A existência ao nível regulamentar, orgânico e pedagógico de medidas promotoras de culturas inclusivas na Universidade Federal de Sergipe e na Universidade da Madeira permite também a discussão comparada de políticas educativas inclusivas sobre o respeito pela vivência e expressões de gênero e sexualidades, bem como uma compreensão ampla das duas sociedades - Brasil e Portugal, historicamente próximas (BRAZÃO; OLIVEIRA; DIAS, 2021).

\section{REFERÊNCIAS}

ALVES, F. C.; FIALHO, L. M. F.; LIMA, M. S. L. Formação em pesquisa para professores da educação básica. Revista Tempos e Espaços em Educação, v. 11, n. 27, p. 285-300, 2018. https://doi.org/10.20952/revtee.v11i27.8582

ALMEIDA, W. R. A. Uniforme escolar e uniformização dos corpos. Revista Tempos e Espaços em Educação, v. 10, n. 22, p. 9-22, 2017.

https://doi.org/10.20952/revtee.v10i22.6134.

Bardin, L. Análise de Conteúdo. Lisboa: Edições 70, 1997.

Bogdan, R.; Bliken S. Investigação qualitativa em educação. Porto: Porto Editora, 2017.

BOGOSSIAN, T. "Já pensou Pedro com um cabelão de Maria Chiquinha! Não combina, né?!”. Geografia e gênero na educação infantil. Revista Tempos e Espaços em Educação, v. 7, n. 12, p. 169-186, 2014. https://doi.org/10.20952/revtee.v0i0.2963

BRAZÃO, J. P. G.; OLIVEIRA, A. L.; DIAS, A. F. (2021). University students' voices on sexual and gender diversity, their relationship with coeducation and pedagogical innovation: a comparative study at the University of Madeira (Portugal) and the Federal University of Sergipe (Brazil). Journal of Research and Knowledge Spreading, v. 2, n. 1, e12445, 2021. https://doi.org/10.20952/jrks2112445

Brazão, P. Apresentação do projeto vozes dos estudantes universitários sobre a diversidade sexual e de género, sua relação com a coeducação e com a inovação pedagógica: um estudo comparativo na Universidade da Madeira e na Universidade Federal de Sergipe. The Brain, 2021. Disponível em: https://bra.in/7vA6Q3 
Brazão, P. (2008). Weblogs, aprendizagem e cultura da escola: um estudo no $1^{\circ}$ Ciclo do Ensino Básico. Tese de Doutoramento apresentada na Universidade da Madeira, 2008. Disponível em: http://hdl.handle.net/10400.13/127

CARDOSO, L. R.; BERTOLDO, T. A. T.; SANTOS, L. B. A. Gênero e sexualidade na formação docente: um mapeamento das pesquisas entre Norte e Nordeste. Revista on line de Política e Gestão Educacional, v. 24, n. esp. 3, p. 1743-1764, 2020. https://doi.org/10.22633/rpge.v24iesp3.14092

CARDOSO, H. M.; RIOS, P. P. S. ; DIAS, A. F. . Professors? representations on gender and sexualities in higher education. REVISTA COCAR (UEPA), v. 13, p. 13-32, 2019.

CARDOSO, H. M. Gênero, sexualidade e escola: contribuições da teorização de Foucault. Revista Tempos e Espaços em Educação, v. 11, n. 1, p. 319-332, 2019. https://doi.org/10.20952/revtee.v11i01.9652

CARDOSO, H. M.; DIAS, A. F. Representações sobre corpo, gênero e sexualidades de estudantes das licenciaturas do Instituto Federal de Sergipe, campus Aracaju. Práxis Educacional, v. 13, n. 24, p. 76-94, 2017. https://doi.org/10.22481/praxis.v13i24.930

CARDOSO, H. M.; DIAS, A. F. Trans* subjectivities in the higher education curriculum. Journal of Research and Knowledge Spreading, v. 2, n. 1, e12305, 2021. https://doi.org/10.20952/jrks2112305

CARVALHO. M. E. P. Et al. Direitos humanos das mulheres e das pessoas LGBTQI: inclusão da perspectiva da diversidade sexual e de gênero na educação e na formação docente. João Pessoa: Editora da UFPB, 2017.

CARVALHO, M. E. P. et al. Origins and challenges of gender studies centers in higher education in NorthERN and Northeastern Brazil. Revista Tempos e Espaços em Educação, v. 10, n. 21, p. 163-176, 2017. https://doi.org/10.20952/revtee.v10i21.6340

CIRQUEIRA, N. S.; SANTANA, J. V. J. de; PEREIRA, R. S. Formação docente e as relações de gênero e sexualidade no curso de Pedagogia. Práxis Educacional, v. 17, n. 45, p. 1-19, 2021. https://doi.org/10.22481/praxisedu.v17i45.8348

COUTO, A. S.; CRUZ, M. H. S. Inserção de gênero no currículo de História e a formação para o trabalho docente. Revista Tempos e Espaços em Educação, v. 10, n. 23, p. 249-262, 2017. https://doi.org/10.20952/revtee.v10i23.6764

DAL'IGNA, M. C.; SCHERER, R. P.; CRUZ, Éderson da. Gênero, sexualidade e formação de professores: uma análise a partir da produção acadêmica da ANPED. Revista Inter Ação, v. 42, n. 3, p. 632-655, 2017. https://doi.org/10.5216/ia.v42i3.48941

DIAS, A. F.; AMORIM, S. Body, gender and sexuality in teacher training: a meta-analysis. Educar em Revista, v. 0, n. 56, p. 193-206. https://doi.org/10.1590/0104-4060.40998

DIAS, A. F.; OLIVEIRA, D. A.; SANTOS, M. S. Uma revisão sistematizada da produção do conhecimento sobre corpo, gênero, sexualidades na educação. Revista Temas em

Educação, v. 27, n. 2, p. 119-133, 2018.

https://doi.org/10.22478/ufpb.2359-7003.2018v27n2.24814 
DIAS, A. F.; SILVA, I. P. ; RIOS, P. P. S. Os estudos de gênero em revistas científicas do FEPAE-NN: uma revisão sistematizada. Revista Exitus, v. 10, n. 1, p. e020039, 2020. https://doi.org/10.24065/2237-9460.2020v10n0ID1287

DIAS, A. F., et al. Schooling and subversions of gender. Revista Tempos e Espaços em Educação, v. 10, n. 22, p. 83-92, 2017. https://doi.org/10.20952/revtee.v10i22.6433

DIAS, A. F.. Corpo, gênero e sexualidades - Problematizando estereótipos (online). RETRATOS DA ESCOLA, v. 9, p. 73-90, 2015.

DONATO, A.; TONELLI, L. A resistência do corpo. Revista Tempos e Espaços em Educação, v. 12, n. 28, p. 49-62, 2019. https://doi.org/10.20952/revtee.v12i28.10164

FRANÇA, F. G. R.; FERRARI, A. Mais do que professores/as, professores/as homossexuais na escola. Revista Tempos e Espaços em Educação, v. 9, n. 20, p. 41-52, 2016.

https://doi.org/10.20952/revtee.v9i20.5894

FERREIRA, M. O. V. O campo do gênero na ANPEd: hipóteses em construção. In: REUNIÃO ANUAL DA ASSOCIAÇÃO NACIONAL DE PÓS-GRADUAÇÃ̃O E PESQUISA EM EDUCAÇÃO - ANPEd, 37, 2015. Florianópolis/ SC. Anais...PNE: tensões e perspectivas para a educação brasileira. Rio de Janeiro: ANPEd, 2015.

FERREIRA, M. O. V.; CORONEL, M. Sobre a legitimação do campo do gênero na ANPEd. Educação e Pesquisa, v. 43, n. 3, p. 815-831, 2017.

FERREIRA, T. de S. Modos de ver, sentir, e questionar: a presença do gênero e da sexualidade no curso de Pedagogia. In: REUNIÃO ANUAL DA ASSOCIAÇÃO NACIONAL DE PÓS-GRADUAÇÃO E PESQUISA EM EDUCAÇÃO - ANPEd, 36, 2013, Goiânia/GO. Anais... Sistema Nacional de Educação e Participação Popular: Desafios para as Políticas Educacionais. Rio de Janeiro: ANPEd, 2013.

GUARANY, A. L. A. ; SANTOS, L. B. A. ; CARDOSO, Lívia de Rezende . Gênero, sexualidade e currículo: mapeamento das pesquisas acadêmicas no Nordeste brasileiro. Cadernos de Gênero e Diversidade, v. 6, p. 421-446, 2020.

GUARANY, A. L. A.; CARDOSO, L. R. (2021). Before the storm, the apparent calm: gender and sexuality in the humanities teacher education curriculums at UFS. Journal of Research and Knowledge Spreading, v. 2, n. 1, e12312, 2021. https://doi.org/10.20952/jrks2112312

JUNQUEIRA, R. A invenção da ideologia de gênero: a emergência de um cenário políticodiscursivo e a elaboração de uma retórica reacionária antigênero. Psicologia Política, v.18, n. 43, p. 449-502. 2018.

KALIL, I. O. Incursões da "ideologia de gênero" na educação. Sur - Revista Internacional de Direitos Humanos, v.16, n. 29, p. 119-128. 2019.

MEDEIROS, M. S.; SANTOS, E. F. Education and work under the perspective of LGBTQIA+ students from the Federal Institute of Sergipe. Journal of Research and

Knowledge Spreading, v. 1, n. 1, e11749, 2020. http://dx.doi.org/10.20952/jrks1111749

MENDONÇA, A.; BRAZÃO, P.; NASCIMENTO, A. M.; FREITAS, D. Estereótipos de género entre os estudantes da formação de professores em educação infantil (0-10 anos): estudo de caso na Universidade da Madeira. Ensaios Pedagógicos, v. 3, n.3, p. 96-106, 2019. 
MENEZES, C. A. A.; DIAS, A. F.; SANTOS, M. de S. What pedagogical innovation does queer pedagogy propose to the school curriculum?. Práxis Educacional, v. 16, n. 37, p. 241258, 2020. https://doi.org/10.22481/praxisedu.v16i37.6168

MORAIS, J. F. S.; BAIÃO, J. C.; DE FREITAS, C. J. Questões de gênero e sexualidade na escola: narrativas docentes. Revista Tempos e Espaços em Educação, v. 13, n. 32, p. 1-15, 2020. https://doi.org/10.20952/revtee.v13i32.11565

MOREIRA, N. R.; EVANGELISTA, N. J.; LOPES DOS SANTOS, J. P. A experiência feminina negra e suas interrogações à política e prática curriculares. Práxis Educacional, v. 15, n. 32, p. 115-131, 2019. https://doi.org/10.22481/praxis.v15i32.5046

MISKOLCI, R.; CAMPANA, M. "Ideologia de gênero": notas para a genealogia de um pânico moral contemporâneo. Revista Sociedade e Estado, v. 32, n. 3, p. 725-747, set.-dez. 2017.

MISKOLCI, Richard. Exorcizando um fantasma: os interesses por trás do combate à ideologia de gênero. Cadernos Pagu, v. 53, e185302, 2018.

NASCIMENTO, L. F.; CAVALCANTE, M. M. D. Abordagem quantitativa na pesquisa em educação: investigações no cotidiano escolar. Revista Tempos e Espaços em Educação, v. 11, n. 25, p. 249-260, 2018. https://doi.org/10.20952/revtee.v11i25.7075

NUNES, C. P. Conversas interativo-provocativas como opção teórico-metodológica nas Ciências Humanas e na educação. Práxis Educacional, v. 16, n. 37, p. 408-439, 2020. https://doi.org/10.22481/praxisedu.v16i37.6207

OLIVEIRA, A. L.; BRAZÃO, J. P. G.; DIAS, A. F. Dialogue about gender, sexuality and bodies in academic context: a possibility of pedagogical innovation? Journal of Research and Knowledge Spreading, v. 2, n. 1, e12484, 2021. https://doi.org/10.20952/jrks2112484

PALMEIRA, L. L. L.; DIAS, A. F. The importance of Teacher education in the face of the perspectives of diversity: in search of an egalitarian society. Journal of Research and Knowledge Spreading, v. 2, n. 1, e12260, 2021. https://doi.org/10.20952/jrks2112260

PIRES, M. A. Gênero e sexualidade nos currículos de formação em Pedagogia na Universidade Federal de Sergipe. Dissertação (mestrado em Educação) - Programa de Pósgraduação em Educação, Universidade Federal de Sergipe. São Cristóvão, p. 180, 2021.

PORTUGAL. D.R. 1. ${ }^{a}$ série N. ${ }^{\circ}$ 97. Resolução do Conselho de Ministros n. ${ }^{\circ}$ 61/2018, de 21 de maio. Estratégia Nacional para a Igualdade e a Não Discriminação 2018-2030 "Portugal + Igual”, XXI Governo Constitucional. 8 de março de 2018. Disponível em: https://dre.pt/application/file/a/115360102

RIOS, P. P. S.; CARDOSO, H. M.; DIAS, A. F. Concepções de gênero e sexualidade d@ $\mathrm{s}$ docentes do curso de licenciatura em pedagogia: por um currículo Queer. Educação \& Formação, v. 3, n. 2, p. 98-117, 2018. https://doi.org/10.25053/redufor.v3i8.272

SANTOS, A. C.; FELDENS, D. G. Vozes do triunfo: narrativas de si de professoras da educação básica. Revista Tempos e Espaços em Educação, v. 11, n. 1, p. 379-392, 2019. https://doi.org/10.20952/revtee.v11i01.9666 
SANTOS, É. S.; LAGE, A. C. Gênero e diversidade sexual na educação básica: um olhar sobre o componente curricular Direitos Humanos e Cidadania da rede de ensino de Pernambuco. Revista Tempos e Espaços em Educação, v. 10, n. 22, p. 69-82, 2017. DOI: https://doi.org/10.20952/revtee.v10i22.6042

SANTOS, M. H. DA S. R.; RIOS, J. A. V. P. Education and cultural differences: boundary educational practices in basic education. Revista Tempos e Espaços em Educação, v. 14, n. 33, p. e13670, 27 fev. 2021. https://doi.org/10.20952/revtee.v14i33.13670

SILVA JUNIOR, P. M.; IVENICKI, A. Entre sexualidades, masculinidades e raça: contribuições do multi/interculturalismo para a prática pedagógica. Revista Tempos e Espaços em Educação, v. 12, n. 29, p. 125-144, 2019.

https://doi.org/10.20952/revtee.v12i29.9326

SILVA, I. P. da; DIAS, A. F.; RIOS, P. P. S. Os estudos de Gênero na Revista Tempos e Espaços em Educação: uma Revisão Sistematizada. Educação \& Formação, v. 5, n. 14, p. 150-175, 2020. https://doi.org/10.25053/redufor.v5i14mai/ago.2495

SILVA, T. O.; RIOS, P. P. S. Gender, sexual diversity and field education: "in rural communities many people do not understand and treat it as a disease". Journal of Research and Knowledge Spreading, v. 1, n. 1, e11418, 2020. https://doi.org/10.20952/jrks1111418

Stake, R. A arte da investigação com estudos de caso. Lisboa: Fundação Calouste Gulbenkian, 2009.

\section{SOBRE OS AUTORES:}

\section{Alfrancio Ferreira Dias}

Doutor em Sociologia, Universidade Federal de Sergipe (UFS); Professor do Departamento de Educação e do Programa de Pós-graduação em Educação da Universidade Federal de Sergipe - Brasil; Líder do ConQuer - Grupo de estudos e pesquisas queer e outras epistemologias feministas; Bolsista de Produtividade em Pesquisa do CNPq. Correio eletrônico: diasalfrancio@academico.ufs.br

iD https://orcid.org/0000-0002-5562-0085

\section{José Paulo Gomes Brazão}

Doutor em Educação - Inovação Pedagógica (2008), pela Universidade da Madeira (UMa), Portugal. Professor e Investigador sénior na área científica de Inovação Pedagógica da Universidade da Madeira. Membro Associado do CENTRO DE INVESTIGAÇÃ̃O EM EDUCAÇÃO DA UNIVERSIDADE DA MADEIRA - FCT- PEst-OE/CED/UI4083/2014 Portugal. Membro Associado ConQuer - Grupo de estudos e pesquisas queer e outras epistemologias feministas (UFS). Correio eletrônico: jbrazao@staff.uma.pt

iD https://orcid.org/0000-0003-3575-4366 